Pentek, T., Legner, C. Konsortialforschung zur Entwicklung von

Referenzmodellen für die Digitalisierung von Unternehmen - Erfahrungen aus

dem Datenmanagement. HMD 57, 296-309 (2020).

https://doi.org/10.1365/s40702-020-00590-2

\title{
Konsortialforschung zur Entwicklung von Referenzmodellen für die Digitalisierung von Unternehmen - Erfahrungen aus dem Datenmanagement
}

\section{Referenzmodelle als Orientierungshilfe für die Digitalisierung von Unternehmen}

Die zunehmende Digitalisierung und die Bewältigung der Datenflut stellt viele Unternehmen vor große Herausforderungen. Sie erfordert eine gesamthafte Betrachtung der strategischen, organisatorischen und technischen Fähigkeiten und deren Entwicklung im Rahmen eines Transformationsprozesses. Oft scheitern Digitalisierungsinitiativen bereits daran, dass unterschiedliche Bereiche eines Unternehmens keine gemeinsame Sprache finden und Mitarbeiter mit dem notwendigen Digitalisierungswissen und interdisziplinärem Erfahrungshintergrund fehlen. (Referenz-)Modelle liefern daher eine wichtige Orientierung für die Digitalisierung in Unternehmen. Sie dokumentieren deskriptives und präskriptives Wissen in einem Anwendungsbereich und unterstützen dadurch die Definition von Soll-Zuständen und Transformationspfaden.

Die deutschsprachige Wirtschaftsinformatik sieht Referenzmodelle als wichtige, praxisrelevante Forschungsergebnisse (Vom Brocke 2016; Fettke und Loos 2004). Sie kann auf erfolgreiche Beispiele der Referenzmodellierung mit hoher Nutzung durch Praktiker zurückblicken, wie z.B. die Referenzmodelle für industrielle Geschäftsprozesse (Scheer 1995), das St. Galler Business Engineering-Modell (Österle 1995) oder das Handels-H für betriebliche Informationssysteme im Handel (Becker und Schütte 2004). Die Entwicklung von Referenzmodellen folgt den Prinzipien gestaltungsorientierter Forschung (Design Science Research), für die in den letzten Jahren umfangreiche wissenschaftliche Leitlinien entwickelt wurden (Sein et al. 2011; Gregor und Hevner 2013; Hevner et al. 2004). In Bezug auf die Praxisrelevanz sehen sich die Forscher jedoch verschiedenen Herausforderungen gegenüber:

- $\quad$ Neben dem "expliziten" Wissen, das in Form von wissenschaftlichen Publikationen vorliegt, entsteht bei Digitalisierungsthemen sehr viel Erfahrungswissen durch die Einführung und Nutzung von Technologien im Unternehmen. Um praxisrelevante Forschung zu betreiben, müssen Forscher die Probleme im Anwendungsbereich identifizieren und neben wissenschaftlichen Artefakten auch die von Praktikern entwickelten Lösungen und Konzepte analysieren. Oft fehlt den Forscher hier der direkte Zugang bzw. wenn dieser existiert, ist die Erfassung und Analyse dieses "impliziten" Wissens extrem aufwändig.

- Bei der Entwicklung von Referenzmodellen sollten die Forschungsergebnisse Empfehlungscharakter haben und wiederverwendbar sein. Allerdings wird praxisorientierte Forschung meist in bilateralen Projekten zwischen einem Industriepartner und einer Hochschule organisiert. Die intendierte bzw. faktische Wiederverwendung eines Referenzmodells über das involvierte Unternehmen hinaus ist daher für den Forscher nur schwer zu erreichen und nachzuweisen.

Im diesem Beitrag argumentieren wir, dass sich diese Herausforderungen bei der Entwicklung von Referenzmodellen durch multilaterale, institutionalisierte Zusammenarbeit zwischen Forschung und Praxis adressieren lassen. Am Beispiel des Kompetenzzentrums Corporate Data Quality (CC CDQ) zeigen wir auf, wie Konsortialforschung (Österle und Otto 2010) für den Wissenstransfer und die rigorose Entwicklung von Referenzmodellen rund um zentrale Fragestellungen der Digitalisierung genutzt werden kann. Das CC CDQ wurde 2006 an der Universität St. Gallen als industriefinanziertes Forschungskonsortium gegründet und hat seither umfangreiche Methoden und Konzepte zum unternehmensweiten Datenmanagement in enger Kooperation zwischen Forschung und Praxis entwickelt. Anhand des Data Excellence Models illustrieren wir die iterative (Weiter-)Entwicklung eines Referenzmodelles, an dem 15 Unternehmen sowie Forscher aus drei Universitäten mitgewirkt haben. Aus dem Entwicklungsprozess und den Erfahrungen leiten wir Empfehlungen für die Referenzmodellentwicklung in multilateralen Projekten zwischen Forschung und Praxis ab. 


\section{Konsortialforschung für den multilateralen Wissenstransfer zwischen Forschung und Praxis}

\subsection{Konsortialforschung als gestaltungsorientierter Forschungsansatz}

Konsortialforschung ist ein gestaltungsorientierter Forschungsansatz, der Wissenstransfer durch multilaterale Zusammenarbeit zwischen Forschung und Praxis ermöglicht und aus dem relevante, rigoros gestaltete Artefakte entstehen (Back et al. 2007). Er beruht darauf, dass „Fachleute aus der Praxis Forschern Zugang zu ihrer Wissensbasis gewähren, bei der Spezifikation von Lösungen mitwirken, Artefakte in ihrer Geschäftsumgebung testen und die Forschungsaktivitäten finanzieren“ (Österle und Otto 2010, S. 273). Die Methode zur Konsortialforschung ist über einen Zeitraum von zwanzig Jahren an der Universität St. Gallen im Forschungsprogramm Business Engineering entstanden und wurde zunehmend formalisiert (Österle und Otto 2010). Als gestaltungsorientierte Forschungsmethode expliziert Konsortialforschung bestehende Richtlinien für multilaterale, langfristig angelegte Forschungsprozesse und beschreibt ein praxisorientiertes, wissenschaftlich rigoroses Vorgehen zur Entwicklung von Artefakten, wie z.B. Methoden und Modellen. Die Forschungsmethode (siehe Abbildung 1) beinhaltet ein iteratives Phasenmodell mit vier Kernaktivitäten (Analyse, Gestaltung, Evaluation, Diffusion). Sie fordert eine konzeptionelle Dokumentation des Anwendungsbereichs in Form eines Metamodells, das generalisierbare Konstrukte beinhaltet und damit für alle am Forschungsprozess beteiligten Personen ein einheitliches Verständnis schaffen.

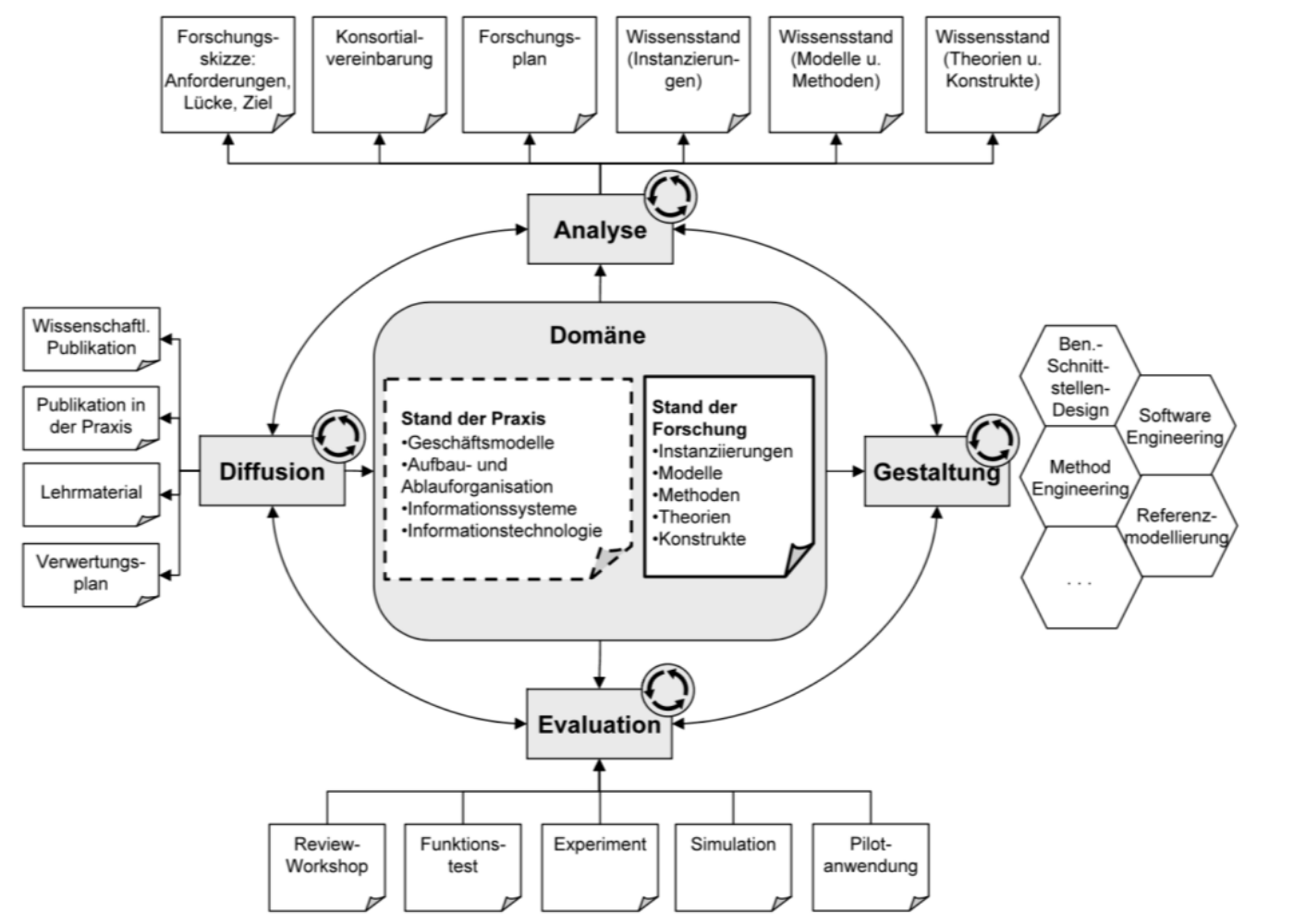

Abbildung 1: Überblick über die Konsortialforschung (Österle und Otto 2010)

\subsection{Konsortialforschung im Kompetenzzentrum Corporate Data Quality (CC CDQ)}

Ein Beispiel für langjährige, erfolgreiche Konsortialforschung ist das CC CDQ, dessen Forschungsergebnisse insbesondere die Referenzmodelle zum unternehmensweiten Datenmanagement - mittlerweile von mehr als einhundert Unternehmen eingesetzt werden. Seit Gründung des CC CDQ im Jahr 2006 haben Wissenschaftler aus drei Universitäten (Universität St. Gallen, TU Dortmund, Universität Lausanne) und mehr als 30 europäische Unternehmen an den Forschungsaktivitäten mitgewirkt. Die Mitgliedsunternehmen stammen aus verschiedenen Branchen wie der Automobil-, Transport, Pharma- oder Konsumgüterindustrie und sind global tätige Großunternehmen mit einem Jahresumsatz von mehr als 1 Milliarde Euro. Grundlage der Forschungsaktivitäten bildet eine Forschungsagenda, in der Forscher und Praxispartner gemeinsam die Forschungsziele und -ergebnisse für das kommende Jahr definieren. Zur Erarbeitung der Forschungsergebnisse hat das CC CDQ seit $2006 \mathrm{mehr}$ als 65 zweitägige Workshops durchgeführt, an denen Firmenvertreter aus dem mittleren Management zentraler Unternehmensfunktionen teilnehmen, z.B. die Leiter Datenmanagement oder Unternehmensarchitekten. Diese beeinflussen und steuern das Datenmanagement in ihrem Unternehmen und bringen damit nicht nur ihr individuelles Expertenwissen ein, sondern repräsentieren ihr Unternehmen, dessen Erfahrungen und Zielsetzungen. Im Rahmen der Workshops sowie begleitender Aktivitäten identifizieren die Forscher 
praxisrelevante Problemstellungen, stellen die von ihnen entwickelten Artefakte vor, analysieren Erfahrungen aus deren praktischer Anwendung und evaluieren diese. Durch mehr als zehn begleitende Dissertationsprojekte sind im Kompetenzzentrum verschiedene Artefakte zu zentralen Fragestellungen des Datenmanagements entstanden. Kernergebnis und Klammer über die Forschungsarbeiten bildet ein Referenzmodell. Dieses entstand in einer ersten Version in den Jahren 2006-2008 als Referenzmodell für das Datenqualitätsmanagement (Corporate Data Quality Management - CDQM) mit Fokus auf Stammdaten (Otto et al. 2011). In den Folgejahren wurden die einzelnen Gestaltungsbereiche des Referenzmodells in weitergehenden Forschungsarbeiten detailliert und auch in praxisorientierten Publikationen veröffentlicht (z.B. Otto et al. 2016). Ausgelöst durch technologische Entwicklungen rund um Big Data und Data Science setzt sich das CC CDQ seit 2012 mit der veränderten Rolle und strategischen Relevanz von Daten und den Implikationen für das Datenmanagement auseinander. Diese Überlegungen führten 2016 zu einer umfassenden Überarbeitung des CDQM Referenzmodelles und dessen Publikation als Data Excellence Model (DXM) (Legner et al. 2020; Pentek et al. 2017).

\section{Entwicklung des Data Excellence Model (DXM) durch Konsortialforschung}

Das DXM kann als wissenschaftlich fundiertes und praxiserprobtes Referenzmodell angesehen werden, das in iterativen Zyklen mit der Praxis entwickelt wurde und dessen Nutzung in zahlreichen Unternehmen - darunter sowohl CC CDQ-Mitglieder wie auch weitere Unternehmen - dokumentiert ist. Der Forschungsprozess zur Entwicklung des Referenzmodells folgte den vier Phasen der Konsortialforschungsmethode, die wir nachfolgend zunächst allgemein beschreiben und dann am Beispiel des DXM illustrieren.

\subsection{Analyse - Anforderungen und Zielsetzung des Data Excellence Models}

\section{Phase:}

Die Analyse-Phase beginnt mit einer ersten, oft vagen Idee zu einem Forschungsthema und dient der Schärfung des Forschungsziels, d.h. des Artefakts und der Anforderungen aus der Praxis. Ausgangspunkt der Entwicklung des DXM waren veränderte und neue Anforderungen an das Datenmanagement in digitalen, datengetriebenen Unternehmen. Vor diesem Hintergrund forderten die CC CDQ Mitgliedsunternehmen Ende 2015, das existierende CDQM Referenzmodell weiterzuentwickeln. Während eines Konsortialworkshops mit 38 Datenmanagementexperten im Februar 2016 wurde zunächst die sich wandelnde Rolle von Daten hin zur strategischen Unternehmensressource und der Umgang mit steigenden Datenanforderungen analysiert. Bei weiteren Workshops im April und Juni 2016 detaillierten 8 bzw. 12 Experten die Anforderungen an das Referenzmodell in Fokusgruppen. Die Anforderungsliste wurde mit akademischer Literatur trianguliert, ergänzt und detailliert.

\section{Ergebnisse:}

Ziel des Referenzmodells sollte es sein, Unternehmen bei der Ist-Analyse und der Ausgestaltung des unternehmensweiten Datenmanagements - als einer zentralen Voraussetzung für die Digitalisierung - zu unterstützen. Wesentliche neue Anforderungen waren die Bewirtschaftung von Daten als strategische Ressource (A1. Geschäftsorientierung) mit entsprechendem Wertbeitrag für das Unternehmen (A5. Wertbeitrag/Nutzen) sowie die Berücksichtigung neuer Datenquellen und des steigenden Datenvolumens (A3. Betrachtungsgegenstand). Neben Datenqualität sollten weitergehende Aspekte wie Datenschutz sowie regulatorische und ethische Prinzipien für den Umgang mit Daten berücksichtigt werden (A4. Datenexzellenz). Da unternehmensweites Datenmanagement verschiedenste Handlungsfelder umfasst, sollte das Referenzmodell - wie bereits das CDQM als die Vorgängerversion - die wichtigsten strategischen, organisatorischen und technologischen Handlungsbereiche adressieren (A2. Gestaltungsbereiche), die in der Praxis nur in einem mehrjährigen Programm schrittweise umgesetzt werden können (A6. Umsetzung). Diese Anforderungen (Tabelle 1) beeinflussten maßgeblich die Gestaltung des Referenzmodells und spiegeln sich in entsprechenden Gestaltungsentscheidungen wider (vgl. Kapitel 3.2).

Tabelle 1: Anforderungen an das Referenzmodell und Gestaltungsentscheidungen

\begin{tabular}{|l|l|l|}
\hline \multicolumn{1}{|c|}{ Anforderung } & \multicolumn{1}{c|}{ Beschreibung } & \multicolumn{1}{c|}{ Gestaltungsentscheidung } \\
\hline $\begin{array}{l}\text { G1. } \\
\text { Geschäftsorientierung2 }\end{array}$ & $\begin{array}{l}\text { Um Daten als strategische Ressource zu } \\
\text { bewirtschaften, muss sich das Datenmanagement auf } \\
\text { die geschäftskritischen Datenanforderungen } \\
\text { ausrichten. }\end{array}$ & $\begin{array}{l}\text { Der Bereich "Ziele" dient der } \\
\text { Übersetzung der } \\
\text { Geschäftsfähigkeiten in } \\
\text { Datenmanagementfähigkeiten. }\end{array}$ \\
\hline $\begin{array}{l}\text { A2. } \\
\text { Gestaltungsbereiche2 }\end{array}$ & $\begin{array}{l}\text { Datenmanagement ist eine soziotechnische } \\
\text { Gestaltungsaufgabe, die strategische, organisatorische } \\
\text { und technologische Anpassungen im Unternehmen } \\
\text { erfordert. }\end{array}$ & $\begin{array}{l}\text { Der Bereich "Enablers" deckt in } \\
\text { seinen Gestaltungsbereichen die } \\
\text { zentralen Handlungsfelder ab. }\end{array}$ \\
\hline
\end{tabular}




\begin{tabular}{|l|l|l|}
\hline $\begin{array}{l}\text { A3. } \\
\text { Betrachtungsgegenstand } 1\end{array}$ & $\begin{array}{l}\text { Vor dem Hintergrund der wachsenden Anzahl von } \\
\text { Datenquellen und Datennutzern sind über Stammdaten } \\
\text { hinaus sämtliche strukturierten Daten (z.B. } \\
\text { Sensordaten) und unstrukturierte Daten (z.B. aus } \\
\text { sozialen Netzwerken) zu berücksichtigen. }\end{array}$ & $\begin{array}{l}\text { Der neue Gestaltungsbereich } \\
\text {,Datenlebenszyklus“ beschreibt } \\
\text { alle relevanten Datenarten und die } \\
\text { operativen Prozesse (z.B. Anlage, } \\
\text { Pflege, Nutzung). }\end{array}$ \\
\hline $\begin{array}{l}\text { A4. } \\
\text { Datenexzellenz } 2\end{array}$ & $\begin{array}{l}\text { Neben der Datenqualität sind weitere Ziele zu } \\
\text { adressieren, wie Datenschutz, Datensicherheit, und die } \\
\text { Einhaltung regulatorischer und ethischer Prinzipien. }\end{array}$ & $\begin{array}{l}\text { Der Bereich "Ergebnisse" umfasst } \\
\text { als datenbezogenes Ergebnis die } \\
\text { Datenexzellenz. }\end{array}$ \\
\hline $\begin{array}{l}\text { A5. } \\
\text { Wertbeitrag / Nutzen } 2 \\
\text { damit auch Datenmanagement einen Wertbeitrag } \\
\text { erzeugen und diesen transparent machen. }\end{array}$ & $\begin{array}{l}\text { Der Bereich "Ergebnisse" umfasst } \\
\text { den Wertbeitrag der Daten und } \\
\text { Nutzen des Datenmanagement. }\end{array}$ \\
\hline $\begin{array}{l}\text { A6. } \\
\text { Umsetzung } 1\end{array}$ & $\begin{array}{l}\text { Der Aufbau von Datenmanagementfähigkeiten kann } \\
\text { nur schrittweise, d.h. im Rahmen längerfristige } \\
\text { Initiativen/Programme, aufgebaut werden. }\end{array}$ & $\begin{array}{l}\text { Datenmanagement folgt einem } \\
\text { Kreislauf zur ,,kontinuierlichen } \\
\text { Verbesserung“. }\end{array}$ \\
\hline $\begin{array}{l}\mathbf{1} \text { Anforderungen an das qualitätsorientierte Datenmanagement, die aus dem CDQM übernommen wurden. } \\
\text { 2Erweiterung der Anforderungen aufgrund der strategischen Bedeutung von Daten und der zunehmenden Datenvielfalt/-menge. }\end{array}$ \\
\hline
\end{tabular}

\subsection{Gestaltung - Aufbau und Inhalte des Data Excellence Models}

\section{Phase:}

In dieser Phase entwickeln Forscher und Praktiker die Artefakte in einem kooperativen, iterativen Prozess. Die Gestaltung des DXM erfolgte während vier Konsortialworkshops im Zeitraum von April 2016 bis Februar 2017. In Fokusgruppen, an denen zwischen 9 und 16 Experten teilnahmen, wurden Gestaltungsoptionen diskutiert, die im Vorfeld der Workshops von den Forschern erarbeitet wurden. In den ersten drei Fokusgruppen diskutierten die Teilnehmer die Struktur, Gestaltungsbereiche, Inhalte und Begriffe des Referenzmodells, während in der vierten Fokusgruppe die grafische Gestaltung im Vordergrund stand. Parallel dazu erfolgte eine Literaturrecherche sowie die Identifikation und Bewertung bestehender Lösungsansätze. Neben theoretischen Ansätzen analysierte das Forschungsteam sowohl die Nutzung des bisherigen CDQM Referenzmodells in den Unternehmen wie auch 11 weitere Referenzmodelle für das Datenmanagement aus der Forschung, von Industriekonsortien, Beratungsunternehmen, Softwareanbietern und Standardisierungsgremien (siehe 4.1).

\section{Ergebnisse:}

Als Referenzmodell für strategisches Datenmanagement beschreibt das DXM die relevanten strategischen, organisatorischen und technologischen Fähigkeiten, um Daten als strategische Ressource zu bewirtschaften und daraus einen Mehrwert für das Unternehmen zu erzielen. Datenmanagement wird entsprechend der wissenschaftlichen Literatur als eine dynamische Fähigkeit (capability) verstanden. Dieses Verständnis von Datenmanagement spiegelt sich in der Struktur des DXM wider:

- $\quad$ Fähigkeiten sind zielorientiert. Das DXM definiert daher im Bereich „Ziele“ (Goals) die relevanten datenbezogenen Geschäftsfähigkeiten (z.B. digitale Geschäftsmodelle, datengetriebene Entscheidungen oder Geschäftsprozesse) und leitet daraus Datenmanagementfähigkeiten ab.

- Fähigkeiten entstehen durch die Kombination von Ressourcen, Routinen und Fertigkeiten. Als sog. Enabler spezifiziert das DXM die soziotechnischen Gestaltungsbereiche, um die benötigten Datenmanagementfähigkeiten aufzubauen. Diese sind weitgehend aus dem CDQM übernommen. Auf organisatorischer Ebene ist das der Gestaltungsbereich „Menschen, Rollen und Verantwortlichkeiten“, auf prozessualer Ebene „Prozesse und Methoden“ sowie der neu eingeführte „Datenlebenszyklus“, auf technologischer Ebene „Datenapplikationen“ und „Datenarchitektur“ und auf steuernder Ebene „Performance Management“.

- Fähigkeiten sind ergebnisorientiert. Der Bereich „Ergebnisse“ (Results) des DXM beschreibt, wie das Unternehmen durch exzellente Daten Geschäftsnutzen erzielt. Diese sog. Monetarisierung von Daten kann durch verbesserte Geschäftsprozesse und Entscheidungen bzw. veränderte oder neue Geschäftsmodelle erfolgen.

- Fähigkeiten sind dynamisch und entwickeln sich weiter. Entsprechend sind Ziele, Enabler und Ergebnisse des Datenmanagements durch einen kontinuierlichen Verbesserungsprozess miteinander verbunden.

Abbildung 2 zeigt das DXM und seine zwölf Gestaltungsbereiche, die den Bereichen Goals, Enablers oder Results zugeordnet sind. Jeder Gestaltungsbereich wird durch die zu gestaltenden Objekte, die Designprinzipien und Ergebnisdokumente weiter spezifiziert. Ein Metamodell definiert die wichtigsten Konstrukte (Objekte) des Datenmanagement und deren Beziehungen.

1 Eine Beschreibung des DXM ist unter https://www.cc-cdq.ch/data-excellence-model zu finden. 


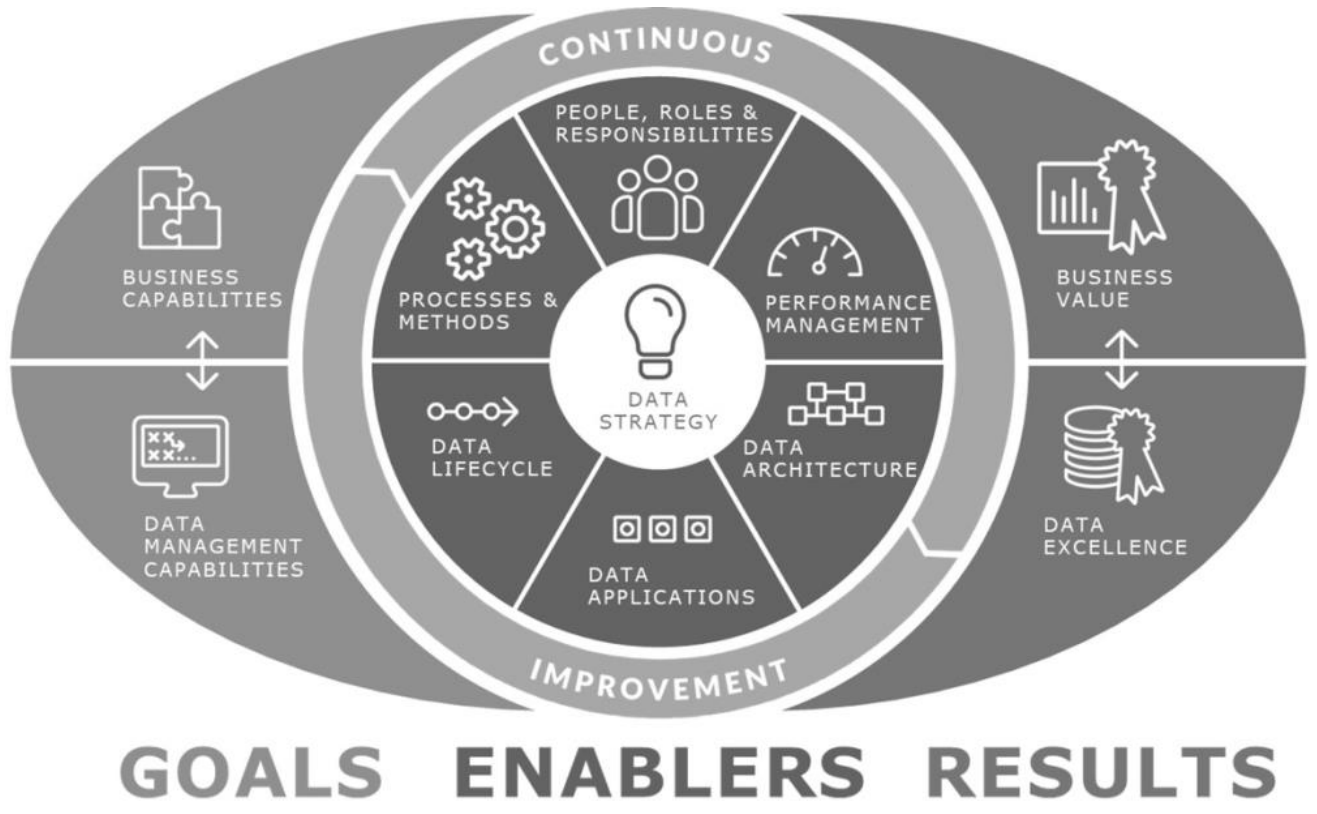

Abbildung 2: Data Excellence Model

\subsection{Evaluation - kontinuierliche und formale Bewertung}

Phase:

In dieser Phase werden die Artefakte kontinuierlich evaluiert und durch Pilotanwendungen hinsichtlich ihrer Anwendbarkeit und ihres Nutzenbeitrags getestet. Die Evaluation des DXM erfolgte kontinuierlich durch die Diskussion von Zwischenständen und Gestaltungsentscheidungen in Fokusgruppen, sowie durch die konkrete Anwendung des Referenzmodells in Piloten und durch formale Bewertungsmethoden.

\section{Ergebnisse:}

Einerseits konnte das Forscherteam analytisch belegen, dass die Anforderungen an das Referenzmodell mit den getroffenen Gestaltungsentscheidungen adressiert werden (vgl. Tabelle 1). Andererseits führte das Forscherteam eine formale, fragebogenbasierte Bewertung bei 25 erfahrenen Datenmanagern durch. Diese orientierte sich an den Bewertungskriterien von Prat et al. (2015) und beinhaltete Fragen zur Struktur, Anpassungsfähigkeit und Anschlussfähigkeit des Referenzmodells. Dabei bewerteten $86 \%$ der Befragten das Referenzmodell als hilfreich für ihre Datenmanagementaktivitäten. Die Mehrheit bestätigte, dass das Referenzmodell alle relevanten Bereiche im Datenmanagement abdeckt (88\%), die Realität angemessen abbildet (83\%) und ausreichend robust ist, um zukünftige Änderungen abzudecken (80\%). Die relativ niedrige Zustimmung (48\%) zur grafischen Repräsentation des Referenzmodells führte zu einer anschließenden Überarbeitung der visuellen Aufbereitung.

\subsection{Diffusion - Aufbereitung für unterschiedliche Zielgruppen und Nutzung des DXM}

\section{Phase:}

In dieser Phase werden die Forschungsergebnisse publiziert und damit allgemein verfügbar gemacht. Das DXM wurde einerseits durch die Veröffentlichung wissenschaftlicher Publikationen und einer Dissertation verbreitet, andererseits in Schulungsunterlagen eines sechstägigen Weiterbildungsprogramms für Datenmanager genutzt. Darüber hinaus ist das DXM Grundlage für ein Reifegradbewertungsmodell, mit dessen Hilfe Unternehmen den Stand ihrer Datenmanagementaktivitäten bestimmen und mit anderen Unternehmen vergleichen können.

\section{Ergebnisse:}

Das DXM wird heute von einem Großteil der CC CDQ Mitgliedsunternehmen sowie von weiteren Unternehmen zur Strukturierung, Weiterentwicklung und Kommunikation ihrer Datenmanagementaktivitäten angewandt. Auch wenn keine Transparenz über sämtliche Unternehmen, die das DXM einsetzen, existiert, so lassen sich anhand der bekannten Fälle typische Einsatzbereiche aufzeigen: (1) die unternehmensspezifische Ausprägung durch Instanziierung des Referenzmodells, und (2) die Nutzung des generischen Referenzmodells als Wissensbasis zu Zwecken der Kommunikation und Ausbildung. Diese Beispiele belegen die faktische Wiederverwendbarkeit des DXM und seine Rolle als Referenzmodell. 
(1) Die unternehmensspezifische Ausprägung des Referenzmodells erfolgt in verschiedenen Formen: Teilweise verwenden Unternehmen das DXM als präskriptives Modell, um ein firmenspezifisches Zielbild und Handlungsempfehlungen für das Datenmanagement zu entwickeln. Ein Fallbeispiel dafür ist ein Pharmaunternehmen, das in seiner Firmenrichtlinie zum Datenmanagement explizit auf das DXM Bezug nimmt und jeden Gestaltungsbereich ausprägt. Die Anpassung des Referenzmodells für eine firmenspezifische Ausprägung erfolgt in der Regel durch die Ausprägung und ggf. Umbenennung von Gestaltungsbereichen und die Anpassung der grafischen Gestaltungen (Farben, Symbole), um anschlussfähig an Firmenrichtlinien und den unternehmensspezifischen Sprachgebrauch zu sein. Darüber hinaus nutzen einige Unternehmen das DXM eher deskriptiv und teilweise gekoppelt mit einer Reifegradbewertung, um ihre Ist-Situation zu analysieren und Schwachpunkte aufzuzeigen. In Digitalisierungs- und Dateninitiativen wird das Referenzmodell auch dazu verwendet, die neu entstehenden Datenmanagementfähigkeiten zu erheben und zu analysieren. So analysiert z.B. ein Automobilzulieferer seine Datenmanagementaktivitäten rund um Sensordaten und die Überwachung von Produktionsanlagen anhand des DXM und schafft dadurch ein gemeinschaftliches Verständnis zu den entstehenden Praktiken.

(2) Neben der firmenspezifischen Ausprägung ist das generische Referenzmodell für zahlreiche Unternehmen eine wichtige Wissensbasis, die zur Kommunikation und zur internen Schulung von Mitarbeitern eingesetzt wird Zum Beispiel verwendet ein Konsumgüterhersteller das Referenzmodell als Kommunikationsinstrument im Mitarbeitermagazin und Intranet zusammen mit erklärenden Texten zu den eigenen Datenmanagementaktivitäten. Die Ableitung aus einem Referenzmodell, das von vielen Unternehmen in Zusammenarbeit mit Forschern erarbeitet wurde, schafft eine höhere Legitimität und Vertrauen in die Maßnahmen.

\section{Erkenntnisse für die Entwicklung von Referenzmodellen}

Die Entwicklung des DXM zeigt, dass Konsortialforschung - als multilaterale Form der Zusammenarbeit zwischen Forschung und Praxis - zu Referenzmodellen führt, die sowohl explizites wie auch implizites Wissen nutzen und sich durch eine hohe praktische Anwendbarkeit auszeichnen. Der Vergleich mit alternativen Referenzmodellen im Datenmanagement erlaubt es, den Beitrag der Konsortialforschung auch anhand objektiver Kriterien einzuordnen und zu bewerten. Aus den Erfahrungen lassen sich schließlich Empfehlungen für die Entwicklung von Referenzmodellen in multilateraler Zusammenarbeit zwischen Forschung und Praxis ableiten.

\subsection{Vergleich des DXM mit alternativen Referenzmodellen im Datenmanagement}

Parallel zum DXM und seinem Vorgängermodell, dem CDQM Referenzmodell, haben sowohl Praktiker (Industriekonsortien, Beratungsunternehmen, Softwareanbietern und Standardisierungsgremien) wie auch Forscher insgesamt 11 weitere Referenzmodelle, Frameworks oder Reifegradmodelle für das Datenmanagement veröffentlicht. In der Praxis besonders verbreitet sind die von Industriekonsortien entwickelten Modelle, wie das DAMA-DMBOK Framework der Data Management Association (DAMA 2017), das Data Quality Framework von GS1 (GS1 2010) und das Data Capability Assessment Model des EDM Councils (EDM Council 2018). Lediglich zwei dieser 11 Referenzmodelle sind von Wissenschaftlern auf Basis theoretischer Grundlagen entwickelt worden, allerdings integriert nur das DXM sowohl die theoretische wie auch die praktische Wissensbasis. Alle 11 Modelle adressieren ähnliche Gestaltungsbereiche wie das DMX, jedoch mit unterschiedlichen Schwerpunkten und Abgrenzungen. So betonen einige stärker die organisatorischen Aspekte und Data Governance, während andere die Datenarchitektur und Systeme in den Vordergrund stellen. Lediglich ein Modell (das DAMA-DMBOK Framework) ist ähnlich umfangreich wie das DXM.

Vergleicht man die konkurrierenden Modelle mit dem DXM so lassen sich interessante Rückschlüsse auf den Beitrag von Konsortialforschung ziehen: Neben dem DXM wurden nur zwei dieser Referenzmodelle (DAMA 2017; Gartner 2014) in mehreren Versionen veröffentlicht. Alle anderen Modelle wurden (bisher) nur einmalig publiziert. Dies steht im Gegensatz zur Entwicklung des Datenmanagements in der Praxis und birgt das Risiko, dass jedes Modell die jeweils aktuellen Grundlagen zwar aufbereitet, aber diese nicht weiterentwickelt und dadurch wichtige Erkenntnisse verloren gehen. Zu den Ausnahmen zählt das DAMA-DMBOK Framework, das Big Data Management in der letzten Version aufgenommen hat, allerdings als separates Kapitel und nicht integriert in die Ansätze des traditionellen Datenmanagements.

Somit ist festzuhalten, dass der Konsortialforschungsansatz im Fall des DXM einen recht einzigartigen Beitrag für Praxis und Wissenschaft bietet: das Referenzmodell ist in einem rigorosen Forschungsprozess (mit iterativer Entwicklung über Fokusgruppen und Evaluierung durch firmenspezifische Instanziierungen) entstanden, der Anforderungen und Erfahrungen aus der Praxis mit theoretischen Konzepte aus der Wissenschaft verbindet und Wissen zum Datenmanagement über einen Zeitraum von mehr als 10 Jahren weiterentwickelt. Insbesondere die Wissensakkumulation und Kontinuität stellen Besonderheiten von Konsortialforschung dar, die zu einer weiten Nutzung und großer Akzeptanz des Referenzmodells in der Praxis beigetragen haben. 


\subsection{Empfehlungen für die Referenzmodellentwicklung in Zusammenarbeit zwischen Forschung und Praxis}

Auf Basis der Erfahrungen bei der Entwicklung des DXM lassen sich Empfehlungen für die Entwicklung von Referenzmodellen in Zusammenarbeit zwischen Forschern und Praktikern ableiten (vgl. Tabelle 2):

- Themengebiet: Wie das Beispiel DXM zeigt, eignet sich Konsortialforschung für interdisziplinäre Problemstellungen im Rahmen der Digitalisierung von Unternehmen, die für mehrere Unternehmen relevant sind und zu denen noch keine reife Wissensbasis vorliegt.

- Projektorganisation: Das DXM wurde über einen Zeitraum von 18 Monaten in einem institutionalisierten, multilateralen Konsortium mit einem stabilen Kern an Praktikern und Forschern entwickelt und durch praktische Anwendung getestet. Im Vergleich zu anderen, meist bilateralen Formen der Zusammenarbeit zwischen Industrie und Forschung, fördern langfristige Strukturen und eine Projektorganisation für die multilaterale Zusammenarbeit - wie im Rahmen der Konsortialforschung - das gegenseitige Vertrauen und Verständnis. Sie erhöhen auch die Qualität und Robustheit der Ergebnisse.

- Forschungsergebnisse: Im Gegensatz zu bilateralen Forschungsprojekten oder Auftragsforschung stehen nicht unternehmensspezifische Ergebnisse im Vordergrund, sondern eine Forschungsagenda mit (allgemeinen) Forschungsergebnissen von hoher wissenschaftlicher und praktischer Relevanz, die zwischen Unternehmen und dem Forscherteam abgestimmt ist. Das Referenzmodell - als zentrales Forschungsergebnis - strukturiert die strategischen, organisatorischen und technischen Fragestellungen und Gestaltungsbereiche im Datenmanagement und bietet damit eine wichtige Orientierungshilfe. Auch wenn die Unternehmen die wissenschaftliche Veröffentlichung des Referenzmodells und die Rigorosität im Forschungsprozess schätzten, müssen die Forschungsergebnisse für Praktiker und Wissenschaftler jeweils zielgruppenspezifisch aufbereitet und kommuniziert werden. Für die praktische Anwendung sind Schulungsmaterialien und Templates von großer Bedeutung.

- Forschungsprozess: Der Forscher ist nicht nur in seiner Rolle als Wissenschaftler, sondern auch als Moderator eines komplexen Forschungsprozesses gefordert. In den frühen Phasen des Forschungsprozesses gilt es aus vagen und teilweise widersprüchlichen Erwartungen an die Forschungsergebnisse gemeinsame Forschungsfragen, -ziele und Anforderungen an das Artefakt zu entwickeln. In der Gestaltungsphase können Forscher durch Analyse und Vergleich bestehender Praxisansätze allgemein gültige Gestaltungsprinzipien ableiten. Die zentralen Konstrukte des Metamodells und die Gestaltungsbereiche des Referenzmodells schaffen eine ontologische Basis und erleichtern die Verständigung zwischen Vertretern unterschiedlicher Disziplinen und Unternehmen. Durch begleitende Evaluation innerhalb des Konsortiums, aber auch mit externen Experten wird die iterative Entwicklung und Qualität des Artefakts gefördert. Die Diffusion für Wissenschaft und Praxis ist gezielt zu planen, denn gerade Praktiker benötigen für die Anwendung eines Referenzmodelles einfache Beschreibungen sowie zusätzliche Hilfestellungen wie z.B. das Reifegradmodell.

Tabelle 2: Erfahrungen und Empfehlungen für die Referenzmodellentwicklung in Konsortialforschungsprojekten

\begin{tabular}{|c|c|c|}
\hline \multicolumn{2}{|r|}{ Erfahrungen bei der Entwicklung des DXM } & \multirow[t]{2}{*}{ Empfehlungen } \\
\hline Fe & & \\
\hline Thema & $\begin{array}{l}\text { Datenmanagement ist ein interdisziplinäres Thema und } \\
\text { sowohl in der Forschung als auch in der Praxis nicht } \\
\text { hinreichend durchdrungen. Technologische und } \\
\text { Marktentwicklungen schaffen neue Herausforderungen und } \\
\text { motivieren Unternehmen zur Zusammenarbeit. }\end{array}$ & $\begin{array}{l}\text { Fokussierung auf wichtige, } \\
\text { soziotechnische Fragestellungen rund } \\
\text { um die Digitalisierung, zu denen keine } \\
\text { reife Wissensbasis existiert und die für } \\
\text { mehrere Unternehmen relevant sind }\end{array}$ \\
\hline $\begin{array}{l}\text { Organisation } \\
\text { (Dauer und } \\
\text { Teilnehmer) }\end{array}$ & $\begin{array}{l}\text { Die Entwicklung des DXM über einen Zeitraum von } 18 \\
\text { Monaten mit einem stabilen Teilnehmerkreis schafft hohes } \\
\text { Vertrauen zwischen Forschern und Praktikern, fördert den } \\
\text { Wissenstransfer und damit die Qualität und Robustheit der } \\
\text { Ergebnisse. }\end{array}$ & $\begin{array}{l}\text { Multilaterale, längerfristig angelegte } \\
\text { Projektorganisation mit aktiver } \\
\text { Beteiligung der Unternehmensvertreter } \\
\text { bei der Erarbeitung und Anwendung der } \\
\text { Forschungsergebnisse }\end{array}$ \\
\hline \multirow[t]{2}{*}{ Ergebnisse } & $\begin{array}{l}\text { Das DXM wurde im Rahmen der jährlichen Planung } \\
\text { Forschungsagenda als zentrales Forschungsergebnis } \\
\text { definiert und strukturiert die strategischen, organisatorischen } \\
\text { und technischen Fragestellungen und Gestaltungsbereiche } \\
\text { im Datenmanagement. }\end{array}$ & $\begin{array}{l}\text { Definition des Referenzmodells als } \\
\text { zentrales Forschungsergebnis zur } \\
\text { Strukturierung des Forschungsgebiets }\end{array}$ \\
\hline & $\begin{array}{l}\text { Als rigoros entwickeltes, praxisrelevantes } \\
\text { Forschungsergebnis muss das DXM für Praktiker und } \\
\text { Wissenschaftler jeweils spezifisch aufbereitet und } \\
\text { kommuniziert werden. }\end{array}$ & $\begin{array}{l}\text { Zielgruppenspezifische Aufbereitung } \\
\text { des Referenzmodells für Praktiker und } \\
\text { für Wissenschaftler }\end{array}$ \\
\hline
\end{tabular}




\begin{tabular}{|c|c|c|}
\hline \multicolumn{3}{|c|}{ Forschungsprozess } \\
\hline Analyse & $\begin{array}{l}\text { Die Teilnehmer starten mit einer vagen Vorstellung der } \\
\text { Ergebnisse und unterschiedliche Erwartungen an die } \\
\text { Forschungsaktivitäten, die einen hohen } \\
\text { Koordinationsaufwand erzeugen. }\end{array}$ & $\begin{array}{l}\text { Frühzeitige Definition und } \\
\text { Dokumentation der Forschungsfragen, } \\
\text {-ziele und Anforderungen an das } \\
\text { Referenzmodell }\end{array}$ \\
\hline \multirow[t]{4}{*}{ Gestaltung } & $\begin{array}{l}\text { Durch Analyse und Bewertung von bestehenden } \\
\text { Lösungsansätzen oder Instanziierungen wurde wichtiges } \\
\text { "implizites" Gestaltungswissen expliziert. }\end{array}$ & $\begin{array}{l}\text { Analyse bestehender Praxisansätze für } \\
\text { die Identifikation von allgemein gültigen } \\
\text { Gestaltungsprinzipien }\end{array}$ \\
\hline & $\begin{array}{l}\text { Verschiedene Unternehmen und Disziplinen verwenden oft } \\
\text { ihre jeweils eigene Sprache. Die zentralen Konstrukte des } \\
\text { Metamodells und die Gestaltungsbereiche des } \\
\text { Referenzmodells erleichtern die Verständigung. }\end{array}$ & $\begin{array}{l}\text { Definition des Metamodells und der } \\
\text { Gestaltungsbereiche des } \\
\text { Referenzmodells als ontologische Basis }\end{array}$ \\
\hline & $\begin{array}{l}\text { Je nach Art der Gestaltungsentscheidung (d.h. Grundsatz- } \\
\text { oder Detailentscheid) nutzte das Forscherteam für einen } \\
\text { effizienten Gestaltungsprozess unterschiedliche } \\
\text { Ansprechpartner und Techniken. }\end{array}$ & $\begin{array}{l}\text { Nutzung unterschiedlicher Techniken: } \\
\text { z.B. Verabschiedung von grundlegenden } \\
\text { Designentscheidungen im Plenum; } \\
\text { Fokusgruppen und Experteninterviews } \\
\text { zur Ausarbeitung von Details }\end{array}$ \\
\hline & $\begin{array}{l}\text { Veränderungen in der Zusammensetzung der Teilnehmer in } \\
\text { den Workshops erzeugte unterschiedliche Wissensstände } \\
\text { und führte zu wiederholten Diskussionen bereits getroffener } \\
\text { Gestaltungsentscheidungen. }\end{array}$ & $\begin{array}{l}\text { Dokumentation und Kommunikation } \\
\text { der Gestaltungsentscheidungen an alle } \\
\text { am Gestaltungsprozess beteiligten } \\
\text { Personen }\end{array}$ \\
\hline \multirow[t]{2}{*}{ Evaluation } & $\begin{array}{l}\text { Die unterschiedlichen Evaluationstechniken (Fokusgruppen, } \\
\text { fragebogenbasierte Bewertung, Instanziierungen) zeigten } \\
\text { Schwachstellen und Verbesserungsmöglichkeiten für } \\
\text { Zwischenstände des DXM und trugen so erheblich zur } \\
\text { Qualität des Endergebnisses bei. }\end{array}$ & $\begin{array}{l}\text { Nutzung unterschiedlicher } \\
\text { begleitender Evaluationstechniken zur } \\
\text { iterativen Entwicklung und Verbesserung } \\
\text { des Referenzmodells }\end{array}$ \\
\hline & $\begin{array}{l}\text { Durch die langjährige Zusammenarbeit im CC CDQ } \\
\text { entstand eine voreingenommene Sicht auf das } \\
\text { Datenmanagement. Relevante Aspekte wurden dadurch } \\
\text { nicht (vollumfänglich) berücksichtigt und erst durch } \\
\text { Interviews mit externen Experten identifiziert. }\end{array}$ & $\begin{array}{l}\text { Einbindung von externen Experten, die } \\
\text { nicht am Gestaltungsprozess beteiligt } \\
\text { waren und eine neutrale Sicht einbringen }\end{array}$ \\
\hline \multirow[t]{2}{*}{ Diffusion } & $\begin{array}{l}\text { Die beteiligten Unternehmen schätzten die wissenschaftliche } \\
\text { Veröffentlichung des Referenzmodells und die durch den } \\
\text { Peer-Review-Prozess bestätigte Rigorosität des Vorgehens } \\
\text { und Ergebnisses. Dennoch benötigen Unternehmensvertreter } \\
\text { eine praxisorientierte Beschreibung des Referenzmodells, } \\
\text { um dieses anwenden zu können. }\end{array}$ & $\begin{array}{l}\text { Planung der Diffusion über } \\
\text { verschiedene Kanäle für Praktiker und } \\
\text { für Wissenschaftler }\end{array}$ \\
\hline & $\begin{array}{l}\text { Referenzmodelle (wie das hier vorgestellte DXM) sind auf } \\
\text { einer detaillierten Ebene erklärungsbedürftig. Für die } \\
\text { Akzeptanz und Nutzung des DXM sind daher neben dem } \\
\text { Artefakt auch die Grundüberlegungen im } \\
\text { Gestaltungsprozess zu kommunizieren. }\end{array}$ & $\begin{array}{l}\text { Transparenz über die } \\
\text { Gestaltungsprinzipien als Teil der } \\
\text { Dokumentation des Referenzmodells }\end{array}$ \\
\hline
\end{tabular}

Zusammenfassend ist festzuhalten, dass Konsortialforschung einen Rahmen für die rigorose Entwicklung praxisrelevanter Artefakte schafft und sich dadurch insbesondere für die Entwicklung von Referenzmodellen rund um die Digitalisierung von Unternehmen eignet. Durch die multilaterale Zusammenarbeit und den strukturierten, iterativen Forschungsprozess adressiert Konsortialforschung klassische Herausforderungen der gestaltungsorientierten Forschung und erhöht die Qualität und Anwendbarkeit der Ergebnisse. Bisher wird Konsortialforschung vorwiegend in den von der Universität St. Gallen geprägten Kompetenzzentren betrieben (Back et al. 2007; Österle und Otto 2010). Jedoch ist davon auszugehen, dass sich die geschilderten Empfehlungen auch auf andere multilaterale Forschungsprojekte übertragen lassen, die beispielsweise über EU-, Bundes- oder Landesmittel gefördert oder als industriefinanzierte Experten-/Erfahrungsgruppen organisiert sind. 


\section{Literatur}

Back A, Krogh G von, Enkel E (2007) The CC Model as Organizational Design Striving to Combine Relevance and Rigor. Syst Pract Act Res 20:91-103.

Becker J, Schütte R (1996) Handelsinformationssysteme. Verlag Moderne Industrie, Landsberg/Lech

DAMA (2017) DAMA-DMBOK; Data management body of knowledge. Technics Publications, Basking Ridge NJ

EDM Council (2018) Data Capability Assessment Model (DCAM) Overview.

https://cdn.ymaws.com/edmcouncil.org/resource/resmgr/featured_documents/EDMC_DCAM_Overview.pdf.

Zugegriffen: 11. September 2018

Fettke P, Loos P (2004) Referenzmodellierungsforschung. Wirtschaftsinf 46:331-340.

Gartner (2014) The Gartner Enterprise Information Management Framework. Gartner.

https://blogs.gartner.com/andrew_white/files/2016/10/On_site_poster.pdf. Zugegriffen: 12. September 2018

Gregor S, Hevner AR (2013) Positioning and Presenting Design Science Research for Maximum Impact. MIS Quarterly 37:337-355

GS1 (2010) Data Quality Framework; Version 3.0

Hevner AR, March ST, Park J, Ram S (2004) Design Science in Information Systems Research. MIS Quarterly 28:75-105

Legner C, Pentek T, Otto B (2020) Accumulating Design Science Research Knowledge with Reference Models Insights from Data Management. Journal of the Association for Information Systems, forthcoming

Österle H (1995) Business Engineering. Prozeß- und Systementwicklung. Springer, Berlin

Österle H, Otto B (2010) Konsortialforschung. Wirtschaftsinf 52:273-285.

Otto B, Kokemüller J, Weisbecker A, Gizanis D (2011) Stammdatenmanagement; Datenqualität für Geschäftsprozesse. HMD 48:5-16

Otto B, Österle H (2016) Corporate Data Quality. Springer, Berlin

Pentek T, Legner C, Otto B (2017) Towards a Reference Model for Data Management in the Digital Economy. In: Maedche A, Vom Brocke J, Hevner AR (Hrsg) Designing the Digital Transformation: DESRIST 2017 Research in Progress Proceedings of the 12th International Conference on Design Science Research in Information Systems and Technology, S 73-82

Prat N, Comyn-Wattiau I, Akoka J (2015) A Taxonomy of Evaluation Methods for Information Systems Artifacts. Journal of Management Information Systems 32:229-267.

Scheer A-W (1995) Wirtschaftsinformatik; Referenzmodelle für industrielle Geschäftsprozesse. Springer Berlin Heidelberg, Berlin, Heidelberg, s.l.

Sein MK, Henfridsson O, Purao S, Rossi M, Lindgren R (2011) Action Design Research. MIS Quarterly 35:3756

Vom Brocke J (2016) Referenzmodellierung; Gestaltung und Verteilung von Konstruktionsprozessen. Logos, Berlin 